\title{
Clinical implications of the immunity of the ocular surface: the effect of extended contact lens wear
}

\author{
ANNA M. AMBROZIAK ${ }^{1,2}$, PIOTR KRAWCZYK ${ }^{1}$, KAMIL SZULBORSKI', PIOTR SKOPIŃSKI ${ }^{1,3}$ \\ 1Department of Ophthalmology, Medical University of Warsaw, Poland \\ ${ }^{2}$ Department of Physics, University of Warsaw, Poland \\ ${ }^{3}$ Department of Histology and Embryology, Center for Biostructure Research, Medical University of Warsaw, Poland
}

\begin{abstract}
The innate immune system of the eye is similar to other mucosal surfaces. The first tier is passive and consists of several anatomic, physical and chemical barriers that collaborate to prevent infection without inducing inflammation. The second tier is active and consists of cellular and secretory components that together cause acute inflammation aimed at eradicating the pathogen. The defenses confront a considerable challenge and infections become a significant cause of morbidity. The essential function of the ocular surface, epithelia and tears in the first place is to create a great barrier that prevents microbial attachment, killing or at least stopping proliferation of constantly attacking organisms, and provide a detection system that in the case of disruption of the primary innate defenses, can activate the adaptive immunity to provide further help to eliminate offending infectious elements. In this paper we describe and discuss a potential impact and clinical implications of extended contact lens wear on the immunity of the ocular surface emphasizing in particular the influence of pathogens like Pseudomonas aeruginosa, Fusarium or Acanthamoeba.
\end{abstract}

Key words: contact lenses, keratitis, Pseudomonas aeruginosa, Acanthamoeba, Fusarium, TLR, $M y D 88$.

(Centr Eur J Immunol 2013; 38 (2): 260-264)

\section{Introduction}

One of the most emblematic and serious complications of contact lens wear, which potentially results in partial or even a complete loss of vision, is bacterial keratitis involving Pseudomonas aeruginosa. Until recently this visionthreatening disease was associated with trauma or ocular surface dysfunction. However, with an increased number of contact lens wearers, the contact lens use has become one of the most essential predisposing factors for microbial keratitis in general, and Pseudomonas aeruginosa in particular. Gram-positive bacteria are the predominant organisms associated with microbial keratitis $(83 \%$ of all positive cultures) and Gram-negative bacteria account only for $17 \%$ of bacterial keratitis. In the population of contact lens wearers, Pseudomonas aeruginosa is responsible for about $30 \%$ of all bacterial keratitis cases [1-3]. Additionally $80 \%$ of bacterial keratitis caused by Gram-negative bacteria were reported in contact lens wearers. Major pathophysiological effects of contact lens wear include allergic, toxic and mechanical or osmotic ones. One of the most important effects of contact lens use is the induced hypoxia and hypercapnia of the corneas. Beyond all doubt the corneal epithelial cells constitute the first line of defense against invading pathogens.

One of the major mechanisms of this antibacterial resistance is the components of the tear film, e.g. lactoferrin, lysozyme, mucins and defensins. Some recent studies of the innate immune system have suggested that toll-like receptor systems might be engaged in the immune system on the ocular surface [3-5].

Toll-like receptors (TLRs) are a family of innate immune recognition receptors that recognize the structure of invad- 
ing microbes, termed pathogen-associated molecular patterns (PAMPs). A well-known member of this family, TLR4, has been extensively studied in cases of pathogen-mediated host response, and it functions as a primary detector of LPS lipopolysaccharide, a component of Gram-negative bacteria. An inflammatory response is initiated by activation of TLR4, by multiple intracellular events, including the activation of nuclear factor $\kappa \mathrm{B}(\mathrm{NF}-\kappa \mathrm{B})$, which stimulates the formation and release of many proinflammatory mediators and adhesion molecules, such as interleukin-1 (IL-1), IL-6, IL-8, tumor necrosis factor $\alpha$ (TNF- $\alpha)$, or intracellular adhesion molecule 1 (ICAM-1) [6]. Toll-like receptor 4 with cluster of differentiation 14 (CD14) in collaboration with LPS-binding protein (LBP) has the ability to induce immune response against infiltration of Gram-negative bacteria [1, 5]. Hypoxia associated with contact lens wear can alter the expression of TLR4. In an experimental model, the expression of mRNA of TLR4 was decreased in cells cultured under hypoxic conditions. These findings are in agreement with other studies reporting that TLR4 expression was reduced in other types of cells and organs, e.g. pulmonary artery endothelial cells. In other cell types, on the other hand, the expression of TLR4 was up-regulated by hypoxia, e.g. microglia cell line. In some cases, the level of expression of mRNA of TLR4 was not changed, like in cultured dendritic cells. These different results in the expression of TLRs under hypoxic conditions may be caused by various hypoxic exposure times in the different experiments. The differences in the types of cells and organs may be valuable for the different TLR4 expression. But the down-regulated expression of TLRs in the corneal cells under hypoxia is consistent with many in vivo and in vitro studies. The LPS-induced expression of IL-6 and IL-8 and $\mathrm{NF}-\kappa \mathrm{B}$ activation are reduced under hypoxic conditions $[4,5,7]$. When epithelial cells, cultured under hypoxia, were stimulated by LPS derived from Pseudomonas aeruginosa co-incubated with CD14 and LBP, the activation of NF- $\kappa B$ was detected, followed by an expression of IL-6 and IL-8. In conclusion, TLR4 signaling pathway may play a role in cornea epithelial cells. In the experimental model, LPS exposure caused a major decrease in the mRNA expression of IL-6 and IL-8 protein synthesis under hypoxic conditions. In addition, the activation of NF- $\kappa$ B stimulated by LPS exposure was also diminished due to hypoxia. It was reported that cigarette smoke provoking hypoxia reduced TLR4 expression and LPS responsiveness in patients with severe chronic obstructive pulmonary disease. Severity of symptoms were correlated with the level of TLR4 expression. In response to pathogens, inflammation and infection, the expression of TLR4 may play a significant role. The contact lens-induced hypoxia may potentially increase the susceptibility to bacterial keratitis by altering the TLR4 signaling pathways [8-10].

There is a greater risk of infection with overnight or extended lens wear, but precise mechanisms by which the cornea is predisposed to infection remains unknown. Lens damage or spoilage and hypoxia have been implicated.
However, even daily disposable lenses (daily use considerably reduces contamination) and silicone hydrogel contact lenses (with improved oxygen transmissibility) are still associated with cases of severe keratitis. Under physiological conditions, the cornea is resistant to Pseudomonas aeruginosa [10]. Contact lens wear compromises the ocular surface and defenses against pathogen invasion. Since many cellular components of innate and acquired immunity are absent or restricted to conjunctival and limbal sites, the normal cornea is considered a relatively immune-privileged site. This immune privilege is obvious from the high success of corneal allografts [11]. $\beta$-defensins are small cationic antimicrobial peptides with direct antimicrobial activity against Gram-negative bacteria like Pseudomonas aeruginosa [12]. Human $\beta$-defensin 2 (hBD-2) is upregulated in human corneal epithelial cells in response to LPS of Pseudomonas aeruginosa. Human $\beta$-defensin 2 can also be upregulated in human epithelial cells by Staphylococcus aureus, or cytokine stimuli through signaling events involving MAP kinases (JNK and p38) and the NF- $\kappa$ B transcription factor [13]. This hBD-2 upregulation in response to Pseudomonas aeruginosa might be reduced by the tyrosine kinase inhibitor, but the mechanisms of Pseudomonas aeruginosa-mediated upregulation are not fully understood. Lipopolysaccharide of Pseudomonas aeruginosa is involved in hBD-2 mRNA upregulation in the corneal epithelial cells $[14,15]$. The upregulation of defensins in the cornea in response to Pseudomonas aeruginosa has also been reported. Some studies suggest a pathway for hBD-2 upregulation by Pseudomonas aeruginosa antigens involving TLRMyD88-mediated signaling, the stress-activated MAP kinase, JNK, and both AP-1 and NF- $\mathrm{BB}$ transcription factors. These data suggest existence of a common pathway for upregulation of epithelial innate defenses in response to bacterial antigens via TLR-mediated signaling. Suppressive effects of contact lens wear on bacterial-induced JNK activity (NF- $\kappa \mathrm{B}$ activation) suggest that the attenuation of hBD-2 occurs after bifurcation of TLR-MyD88-dependant pathway. A small reduction in TLR2, but not TLR4 or TLR5, expression was noticed in lens-exposed cells in the presence of bacterial antigens. In corneal epithelial cells it has been shown that TLR 2 had been implicated in hBD-2 upregulation in response to a synthetic lipopeptide antigen (Pam3Cys) - analogue of the N-Terminal of Gram-negative bacterial lipoprotein. It is possible that the reduction of the TLR2 contributes to contact lens-mediated hBD-2 upregulation induced by Pseudomonas aeruginosa. The suppressive influence of contact lens on JNK/AP-1 in vitro may suggest the anti-apoptotic effects of contact lenses on corneal epithelium during contact lens wear in vivo $[16,17]$. JNK and c-Jun activation may lead to induction of AP-1dependent genes responsible for apoptosis.

It is proven that AP-1-mediated gene transcription plays a significant role in numerous mammalian cell processes affecting proliferation, migration and the expression of 
cytokines or matrix metalloproteinases, so contact lens suppression of JNK/AP-1 responses could possibly have other effects on the ocular surface epithelium. AP-1 genes are also known for their involvement in cellular responses to hypoxia, an established complication of soft lenses. Contact lenses not only have the ability of inducing hypoxia, but possibly may initiate cellular response to it. Lensinduced mechanical irritation of the ocular surface, hypoxia, or chemical effects of the lens packaging solution, even the lens material itself could each have a role. Nuclear factor $\kappa \mathrm{B}$ is a major mediator of cytokine expression in inflammatory response, phagocyte infiltration and activation, and finally corneal damage due to invasion of bacterial pathogens including Pseudomonas aeruginosa $[18,19]$.

\section{Acanthamoeba}

Acanthamoeba keratitis is a sight-threatening progressive corneal disease, first described by Nagington in the UK. Until early 1980s it remained extremely rare, but due to the growing use of contact lens and poor lens hygiene, it became a more frequent corneal pathology. In the United States, $85 \%$ of patients with Acanthamoeba keratitis were contact lens wearers [20]. Wearing contact lens overnight, not disinfecting the lenses as often as recommended, using tap water to rinse the lenses are the most common risk factors for corneal infection. Macrophages and neutrophils are crucial in resistance to many infectious pathogens. It has been shown that macrophages and neutrophils were chemotactically attracted Acanthamoeba trophozoites, and are capable of killing trophozoites in vitro [21]. In an experimental model, the presence of interferon $\gamma$ and Acanthamoeba-specific antibody increased the capacity of macrophages to eliminate trophozoites. Macrophages and neutrophils are not chemotactically attracted to intact cysts, while both are attracted to cysts being lysed. In comparison with activated macrophages killing amoebae trophozoites, activation of macrophages with lipopolysaccharide and interferon $\gamma$ did not influence their cytolytic effect on cysts. Macrophages kill cysts by phagocytosis and neutrophils through secretion of myeloperoxidase (MPO). An experimental subconjunctival injection of dichloro-methylene diphosphonate (chlodronate), a substance known for its macrophagicidal activity, provoking depletion of conjunctival macrophages, dramatically exacerbated the incidence, severity and chronicity of infection in experimental animals [21]. In another study, an intraperitoneal injection of anti-Chinese hamster neutrophil antibody considerably exacerbated the disease in these animals. An intracorneal injection of latex beads induced remarkable resistance to Acanthamoeba keratitis to a great extent mediated by macrophages. In mammalian cornea in vitro, resolution of Acanthamoeba keratitis occurs with production of macrophages inflammatory protein-2 (MIP-2), a chemotactic factor for neutrophils. In an experimental model, due to the use of anti-MIP-2, selec- tively inhibiting neutrophil migration, corneal disease was more severe in duration and clinical appearance [1, 23].

In many cases, Acanthamoeba keratitis in spite of efficient antiamebial drugs being used, has a strongly progressive and severe character, and in many patients leads in consequence to corneal opacity or perforation. Moreover, patients need to be monitored for recurrence of the disease including post penetrative keratoplasty because of the resistance of the cysts to a variety of drugs and its ability to survive in the corneal tissue for a long time. Cysts might have some potential to suppress innate immunity, apparently macrophages and neutrophils do not work adequately against Acanthamoeba, but still the innate immunity probably is the most crucial factor in resistance to Acanthamoeba keratitis. The proper functioning of innate immunity of the ocular surface depends on cooperation of immunocytes and relative cytokines, which all together form an efficient network. Exogenous IL-1 or TNF- $\alpha$ can stimulate the synthesis of IL- 6 and IL-8, which may be responsible for the recruitment of macrophages and neutrophils on the ocular surface, as mentioned earlier in this paper. The platelet activating factor (PAF) and leukotriene B4 (LTB4) are produced in the corneal tissue in response to hypoxia, injury or infection; all those three might be potentially provoked by contact lens wear. It is proven that extended and improper contact lens wear alter PMNs, cytokines and chemokines. Contact lens creates a barrier for PMNs to recruit and migrate to the site of immune response and delay the initial inflammatory response. Recent studies suggest that the mechanism of recruiting inflammatory cells to corneas in overnight contact lens wearers modify the chemotactic signals or inhibit partially the activation of the recruited cells [24]. Contact lens wear influences also the concentration of inflammatory mediators in the tear film. In contact lens wearers, the levels of IL- 8 are considerably higher in the tear film compared to the non-contact lens group of patients. ELISA showed that the concentration of IL- 8 was over $100 \mathrm{ng} / \mathrm{ml}$ in contact lens wearers particularly during the sleep, the peak chemotactic activity of IL-8 for PMNs is about 70-90 ng/ml, and abnormal concentrations could result in a decreased number of PMNs. Leukotriene B4 alone was not chemotactic, but IL-8 could induce PMN chemotaxis. Combinations of these two - IL-8/LTB4 showed enhanced chemotaxis compared to IL-8 alone. With comparable concentrations of IL-8/LTB4, chemotactic activity for immune cells in contact lens wearers was lower than in non-contact lens wearers [24]. Extended lens wear may influence occurrence and progression of Acanthamoeba keratitis through altering innate immunity of the ocular surface by affecting the response of PMNs and cytokines, declining defense abilities of the cornea against pathogens and increasing the susceptibility to parasitic infection. After Acanthamoeba invasion the cornea of extended contact lens wearers cannot be properly activated and tissue cells may not be able to secrete enough cytokines and chemokines in response to infection. Macrophages and neutrophils are not adequately activated and do not obtain suffi- 
cient signals to differentiation and migration in those patients. Finally, Acanthamoeba trophozoites and cysts cannot be entirely killed or removed. The immunological mechanism of contact lens wear on the Acanthamoeba keratitis may give more contributions to the future therapies of this sight-threatening disease.

\section{Fusarium}

During a recent and well-described outbreak of contact lens-associated fungal keratitis worldwide, the causative organisms were Fusarium solani and Fusarium oxysporum. We will describe fungal keratitis using as a model Fusarium keratitis. Because of many strong analogies and similarities with other types of fungal keratitis, on a molecular and histological level, we consider such description as legitimate. Fusarium keratitis is associated with biofilm formation and contact lens wear. It has a high prevalence in warm, humid areas of the United States, and in southern and southeastern Asia, especially India and China. Fusarium species and other filamentous fungi are an increasing cause of human mycoses. In neutropenic or immune-compromised individuals they cause endophthalmitis, but the most common manifestation of Fusarium infection is keratitis, occurring in healthy subjects. Pathogenesis is based on the traumatic injury facilitating entry of spores into the corneal stroma. Once present in the cornea, germination, toxin secretion and hyphal invasion take place, which initiates a host response provoking extensive inflammatory tissue damage, so that keratitis results from unregulated microbial growth and consequent tissue degradation. Fusarium keratitis is characterized by profound neutrophil infiltrates and subsequent destruction of the cornea, indicating the major role for the host response in controlling fungal growth and development of keratitis. Under the physiological conditions, the normal cornea maintains its transparency because of the barrier function of the external corneal epithelium, highly organized, antiparallel arrangement of collagen fibrils, and a single layer of corneal endothelial cells lining the anterior chamber, which helps to maintain the proper hydratation level. Other factors are the absence of blood vessels and resident inflammatory cells like neutrophils in the corneal stroma. During the microbial infection the cornea becomes opaque after release of cytotoxic mediators from microbes and from activated neutrophils that infiltrate the corneal stroma. Activation of TLR2 and TLR4 through MyD88 in the cornea induces production of proinflammatory and chemotactic cytokines, which subsequently provokes recruitment of neutrophils to the corneal stroma and loss of its transparency. In response to Fusarium, MyD88 plays an essential role in recruitment of neutrophils. Toll-like receptor 2 and TLR4 have no role in this stage of host response, TLR4 has the same function in fungal killing. The observation that TLR4 has a significant role in fungal killing but not in neutrophil recruitment suggests that the expression of TLR4 on neu- trophils may play a role in mediation of antifungal activity, possibly production of reactive oxygen species (ROS). Tolllike receptor 2 and TLR4 are also important in the host response in other filamentous fungi such as Aspergillus fumigatus or Candida albicans. Hyphal and macroconidia forms of the organisms stimulate different TLRs including TLR4 activation. In an experimental model, mice defective in ROS production by neutrophils are highly susceptible to infection with fungal pathogens, and its mechanism is probably involved in limiting Fusarium growth in the cornea and in the anterior chamber [25].

The process of neutrophil recruitment into the stroma is MyD88 dependent, but independent of TLR2 and TLR4. The role of IL-R1 is worth mentioning. It gives signals through $\mathrm{MyD} 88$ and is required for production of CXCL1/KC and neutrophil recruitment. Interleukin 1 and CXC chemokines have also an important role in Pseudomonas aeruginosa keratitis. Interleukin $1 \mathrm{R} 1$ is required for $\mathrm{CXC}$ chemokine, but not IL-1 production in the cornea, indicating that an indentified receptor is needed for IL-1 production in the cornea. Tolllike receptor 4 is involved in fungal killing, but there is a strong probability that other receptors might be involved. The role of C-type lectins and Dectin-1, proteins expressed on macrophages and neutrophils has not been yet elucidated. They both recognize $\beta(1,3)$-glucan on the fungal cell wall, and cooperate probably with TLRs. Probable events leading to fungal keratitis in immune-competent subjects involves recognition of Fusarium by resident cells in the stroma and production of IL- $1 \alpha$, then IL-1R1/MyD88dependent CXC chemokine production and recruitment of neutrophils from peripheral vessels to the cornea, TLR4dependent antifungal activity initiated by neutrophils, tissue damage and development of corneal opacification mainly due to production of cytotoxic mediators by fungi or caused by neutrophil degranulation [26-30].

The tear film, corneal epithelium, palpebral and eyeball conjunctiva as a structural and functional surface exhibit a characteristic immune profile. The specificity of the immune response implies conditions and complications associated with contact lens wear. It is worth emphasizing that the surface of the eye is critical for the safe use of contact lenses. Knowledge about the immunology of the ocular surface and its pathophysiology associated with extended contact lens wear should be an integral part of every ophthalmological practice.

The authors declare no conflict of interest.

\section{References}

1. Dartt DA, Dana R, D'Amore P (2010): Immunology, inflammation and diseases of the eye. Elsevier Ltd, Philadelphia.

2. Stapleton F, Edwards K, Keay L, et al. (2012): Risk factors for moderate and severe microbial keratitis in daily wear contact lens users. Ophthalmology 119: 1516-1521. 
3. Benson WH, Lanier JD (1998): Current diagnosis and treatment of corneal ulcers. Curr Opin Ophthalmol 9: 45-49.

4. Davidson HJ, Kuonen VJ (2004): The tear film and ocular mucins. Vet Ophthalmol 7: 71-77.

5. Huang X, Hazlett LD, Du W, Barrett RP (2006): SIGIRR promotes resistance against Pseudomonas aeruginosa keratitis by down-regulating type-1 immunity and IL-1R1 and TLR4 signaling. J Immunol 177: 548-556.

6. Oleś D, Szczepankiewicz A (2012): Role of T-cell receptors in the development of allergic inflammation in asthma. Postep Derm Alergol 29: 275-278.

7. Hara Y, Shiraishi A, Ohashi Y (2009): Hypoxia-altered signaling pathways of toll-like receptor 4 (TLR4) in human corneal epithelial cells. Mol Vis 15: 2515-2520.

8. Ishida I, Kubo H, Suzuki S, et al. (2002): Hypoxia diminishes toll-like receptor 4 expression through reactive oxygen species generated by mitochondria in endothelial cells. J Immunol 169: 2069-2075.

9. Ock J, Jeong J, Choi WS, et al. (2007): Regulation of Toll-like receptor 4 expression and its signaling by hypoxia in cultured microglia. J Neurosci Res 85: 1989-1995.

10. MacRedmond RE, Greene CM, Dorscheid DR, et al. (2007): Epithelial expression of TLR4 is modulated in COPD and by steroids, salmeterol and cigarette smoke. Respir Res; 8: 84.

11. Ramphal R, McNiece MT, Polack FM (1981): Adherence of Pseudomonas aeruginosa to the injured cornea: a step in the pathogenesis of corneal infections. Ann Ophthalmol 13: 421-5.

12. McNamara NA, Van R, Tuchin OS, Fleiszig SM (1999): Ocular surface epithelia express mRNA for human beta defensin2. Exp Eye Res 69: 483-490.

13. McDermott AM, Redfern RL, Zhang B (2001): Human betadefensin 2 is up-regulated during re-epithelialization of the cornea. Curr Eye Res 22: 64-67.

14. Kumar A, Zhang J, Yu FS (2006): Toll-like receptor 2-mediated expression of beta-defensin-2 in human corneal epithelial cells. Microbes Infect 8: 380-389.

15. McIntosh RS, Cade JE, Al-Abed M, et al. (2005): The spectrum of antimicrobial peptide expression at the ocular surface. Invest Ophthalmol Vis Sci 46: 1379-1385.

16. Lee EJ, Cowell BA, Evans DJ, Fleiszig SM (2003): Contribution of ExsA-regulated factors to corneal infection by cytotoxic and invasive Pseudomonas aeruginosa in a murine scarification model. Invest Ophthalmol Vis Sci 44: 3892-3898.

17. Gaur U, Aggarwal BB (2003): Regulation of proliferation, survival and apoptosis by members of the TNF superfamily. Biochem Pharmacol 66: 1403-1408.

18. Mastyugin V, Mezentsev A, Zhang WX, et al. (2004): Promoter activity and regulation of the corneal CYP4B1 gene by hypoxia. J Cell Biochem 91: 1218-1238.

19. Kernacki KA, Goebel DJ, Poosch MS, Hazlett LD (1998): Early TIMP gene expression after corneal infection with Pseudomonas aeruginosa. Invest Ophthalmol Vis Sci 39: 331-335.

20. Seal DV (2003): Acanthamoeba keratitis update-incidence, molecular epidemiology and new drugs for treatment. Eye (Lond) 17: 893-905.

21. Larkin DF, Easty DL (1991): Experimental Acanthamoeba keratitis: II. Immunohistochemical evaluation. Br J Ophthalmol 75: 421-424.

22. van Klink F, Taylor WM, Alizadeh H, et al. (1996): The role of macrophages in Acanthamoeba keratitis. Invest Ophthalmol Vis Sci 37: 1271-1281.
23. Lawin-Brüssel CA, Refojo MF, Leong FL, Kenyon KR (1995): Scanning electron microscopy of the early host inflammatory response in experimental Pseudomonas keratitis and contact lens wear. Cornea 14: 355-359.

24. Tarabishy AB, Aldabagh B, Sun Y, et al. (2008): MyD88 regulation of Fusarium keratitis is dependent on TLR4 and IL-1R1 but not TLR2. J Immunol 181: 593-600.

25. Bellocchio S, Moretti S, Perruccio K, et al. (2004): TLRs govern neutrophil activity in aspergillosis. J Immunol 173: 74067415.

26. Lavigne LM, Albina JE, Reichner JS (2006): Beta-glucan is a fungal determinant for adhesion-dependent human neutrophil functions. J Immunol 177: 8667-8675.

27. Ambroziak AM (2007): Influence of the continuous silicone hydrogel contact lens wear on the corneal physiology with special focus on the tear film evaluation. Kontaktologia i Optyka Okulistyczna 2: 13-19.

28. Wójcik-Gryciuk A, Ambroziak AM (2007): Acanthamoeba keratitis in contact lens users. Kontaktologia i Optyka Okulistyczna 1: 40-42.

29. Viriyakosol S, Fierer J, Brown GD, Kirkland TN (2005): Innate immunity to the pathogenic fungus Coccidioides posadasii is dependent on Toll-like receptor 2 and Dectin-1. Infect Immun 73: 1553-1560. 